\title{
The Isomer Effect on Complex Formation in Syndiotactic Polystyrene-Xylene System
}

\author{
Kimiaki Tsutsui, Toshiyuki Katsumata, Hiroki Fukatsu, Hiroaki Yoshimizu, ${ }^{\dagger}$ \\ Takatoshi Kinoshita, and Yoshiharu TsujITA
}

Department of Materials Science and Engineering, Polymeric Materials Course, Nagova Institute of Technology, Gokiso-cho, Showa-ku, Nagoya 466-8555, Japan

(Received August 21, 1998)

\begin{abstract}
Thermal behaviors of syndiotactic polystyrene (SPS) gels in SPS-xylene isomer system were investigated to get information on the isomer effect of xylene on ability of complex formation with SPS. The melting temperature of the crystalline complex of $p$-xylene with SPS in SPS- $p$-xylene system was higher than that in SPS $m$-xylene system. The weight of the gel sample in each thermogravimetric curve decreased by two step in both systems. This might suggest that there were two spaces in which xylene molecules could be located. The weight of $p$-xylene in the second step decreased more largely and at higher temperature than that of $m$-xylene. We could conclude that $p$-xylene exhibited higher ability of complex formation with SPS than $m$-xylene from thermal analyses. Infrared results on the process of gelation showed that the content of TTGG conformation was larger and the completion of gelation was faster in SPS- $p$-xylene system. The difference of sorption behaviors at $25 \mathrm{C}$ of the annealed atactic polystyrene film were not observed between xylene isomers, but the $p$ - and $m$-xylene sorption isotherms of the annealed SPS film showed sigmoid-type isotherms and were different between xylene isomers. Sorption amount of $p$-xylene was larger than that of $m$-xylene at low activity, but smaller at high activity, which resulted in the preferential sorption of $p$-xylene into a mesophase at low activity and the decrease of an amorphous part by complex formation of $p$-xylene with SPS at high activity.

KEY WORDS Syndiotactic Polystyrene / Xylene Isomers / Xylene Vapor Sorption / $\delta$ Form(Crystalline Complex) / Mesophase / Plasticization/
\end{abstract}

Crystalline complexes of polymers with organic molecules have been investigated for separating isomers, preparing highly stereoregular polymers, and obtaining monodispersed fractions from unfractionated polymer samples for many years. ${ }^{1-6}$ The recently synthesized highly syndiotactic polystyrene (SPS) is characterized by complex formation with various organic molecules. ${ }^{7-11}$ The complex with organic molecules is called $\delta$ form, in which SPS chains exhibit TTGG helical conformation. ${ }^{12-15}$ In SPS- organic molecule system, the guest molecules are housed between the cavities generated by two adjacent phenyl groups whose position and distance are given by TTGG helical conformation. SPS always exhibits TTGG helix when it complexes with organic molecules because of the stiff chains. Accordingly, it may be expected that organic solvents whose molecular size and shape fit the cavities formed by two adjacent phenyl groups of SPS chains show high ability of complex formation with SPS.

We will report the isomer effect on complex formation in SPS-xylene system in this article. Complex formation of xylene isomers with SPS in solution system was investigated by using thermal and spectroscopic analyses. For investigation of complex formation in SPS film-vapor system, the xylene isomer sorption behaviors of SPS films were also reported. The sorption behaviors in SPS-organic vapor system are expected to be very complex due to complex formation of xylene isomers with SPS, different from the system in which penetrant molecules are excluded from the crystal lattice when crystallization occurs. So, it is expected that the sorption behaviors in SPS-xylene vapor system may be affected by the difference of ability of complex formation

\footnotetext{
${ }^{\dagger}$ To whom correspondence should be addressed.
}

with SPS between xylene isomers. Recently, it was reported that the structural organization in SPS film induced by toluene vapor sorption and the preferential sorption in a mesophase, which is defined as the phase which does not have a crystalline regularity but a conformational order. ${ }^{16,17}$ We suggested the preferential sorption in the mesophase from the concavity of the toluene sorption isotherm against the activity axis at low activity. Taking the formation mechanism of the mesophase into consideration, it is possible that the sorption sites in the mesophase can memorize molecular size and shape of the cast solvents. So, it is also investigated whether the mesophase can memorize molecular size and shape of the cast solvents.

\section{EXPERIMENTAL}

SPS was kindly supplied by Idemitsu Petrochemical Co.Ltd. and was used without further purification. The weight-averaged molecular weight is $M_{w}=211000$ $\left(M_{w} / M_{n}=3.45\right)$. Atactic polystyrene (APS) was purchased by Aldrich Chem. Co., Inc. and the weightaveraged molecular weight is $M_{w}=280000$. Xylene isomers ( $p$ - and $m$-xylene; described as PX and MX, respectively) were purchased from Nacalai Tesque.Inc. and used without further purification. $10 \mathrm{wt} \%$ SPS was dissolved in PX or MX at the boiling point of PX or MX. SPS gels were prepared by cooling the solution to room temperature. The SPS film prepared by casting from the PX solution at $90^{\circ} \mathrm{C}$ was annealed at $140^{\circ} \mathrm{C}$ for $1 \mathrm{~h}$ in vacuum (described as 1 PX140). The APS film prepared by casting from the PX solution at $90^{\circ} \mathrm{C}$ was also annealed at $140^{\circ} \mathrm{C}$ for $1 \mathrm{~h}$ in vacuum (described as 1PX140(APS)). Thermal analyses and IR measusements showed that there were no solvents in both samples. 
Table I. Physical constants for xylene isomers

\begin{tabular}{lcc}
\hline \multicolumn{1}{c}{ Parameter } & \multicolumn{2}{c}{ Isomer } \\
\cline { 2 - 3 } & $p$-Xylene & $m$-Xylene \\
\hline $\mathrm{bp} /{ }^{\circ} \mathrm{C}$ & 138.4 & 139.1 \\
$d / \mathrm{g} \mathrm{cm}^{-3}$ & 0.8611 & 0.8642 \\
$V D W V / \mathrm{cm}^{3} \mathrm{~mol}^{-1}$ & 70.66 & 70.66 \\
$m_{\mathrm{v}} / \mathrm{cm}^{3} \mathrm{~mol}^{-1}$ & 123.8 & 123.4 \\
\hline
\end{tabular}

Abbreviations are as follows: bp, boiling point ${ }^{20} ; d$, density between 4 and $20^{\circ} \mathrm{C}^{21} ; V D W V$, van der Waals volume ${ }^{20} ; m_{\mathrm{v}}$, molar liquid volume at $25^{\circ} \mathrm{C} .{ }^{20}$

Differential scanning calorimetry (DSC) thermograms of SPS gels in the range $25-150^{\circ} \mathrm{C}$ were obtained using a Perkin Elmer DSC 7 at a heating rate of $2^{\circ} \mathrm{C} \mathrm{min}-1$ under a nitrogen gas purge. Thermogravimetric analysis (TG) data of SPS gels in the range $25-300^{\circ} \mathrm{C}$ were obtained using a Seiko TG/DTA220 at a heating rate of $2^{\circ} \mathrm{C} \mathrm{min}^{-1}$ under a nitrogen gas purge. Infrared (IR) absorbances characteristic of TTGG conformation in SPS gels were evaluated by ATR-FT-IR measurement. IR spectra of SPS gels were obtained using a Perkin Elmer FT-IR spectrometer Spectrum 2000 with $1 \mathrm{~cm}^{-1}$ resolution in the range $2000-700 \mathrm{~cm}^{-1}$ at room temperature and the number of accumulation cycles was 4. The ATR plate was made of $\mathrm{ZnSe}$ crystal. Timeresolved FT-IR measurement was started as soon as SPS solution was poured into the ATR trough. The obtained spectra were normalized by the peak intensity at $1183 \mathrm{~cm}^{-1}$ as an internal standard.

PX and MX vapor sorption isotherms at $25^{\circ} \mathrm{C}$ were obtained by Quartz Crystal Microbalance (QCM) method. QCM has become a popular method for detection of a very small amount of weight because its resonance frequency can sensitively decrease upon the increase of mass on the QCM in the nanogram level. ${ }^{18}$ The thin films were coated on the electrode on quartz crystal oscillator by spin-casting and annealed under the appropriate conditions. Sorption amount of toluene vapor (C $\mathrm{mol} /$ styrene unit $\mathrm{mol}$ ) is plotted against activity $\left(p / p_{0}\right) ; p$ and $p_{0}$ are actual and saturated pressure of toluene vapor at $25^{\circ} \mathrm{C}$, respectively.

\section{RESULTS AND DISCUSSION}

\section{Isomer Effect on Complex Formation of Xylene Isomers with SPS}

The thermoreversible gels in SPS-organic solvent system consist of crystalline complexes of SPS with the organic solvents, which are housed between cavities generated by two adjacent phenyl groups of SPS chains. SPS always exhibits TTGG conformation when it complexes with organic solvents. Accordingly, we can expect the different complex formation with SPS between xylene isomers because their molecular size and shape are different, respectively, although physical properties are almost identical (Table I).

Figure 1 shows DSC thermograms of $10 \mathrm{wt} \%$ SPSxylene gels. An endothermal peak is observed in each thermogram, and the peak temperature for PX is higher than that for MX. The peaks observed in both thermograms are assigned to the desorption of complexed

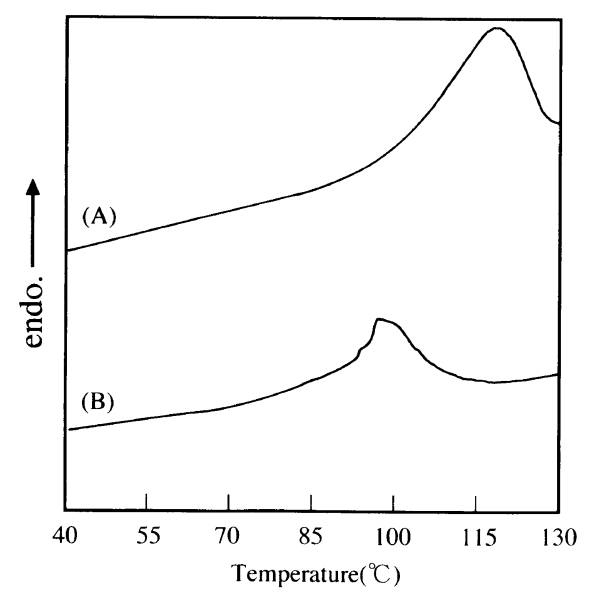

Figure 1. DSC thermograms of SPS-xylene isomer gels: (A) $p$-xylene and (B) $m$-xylene.

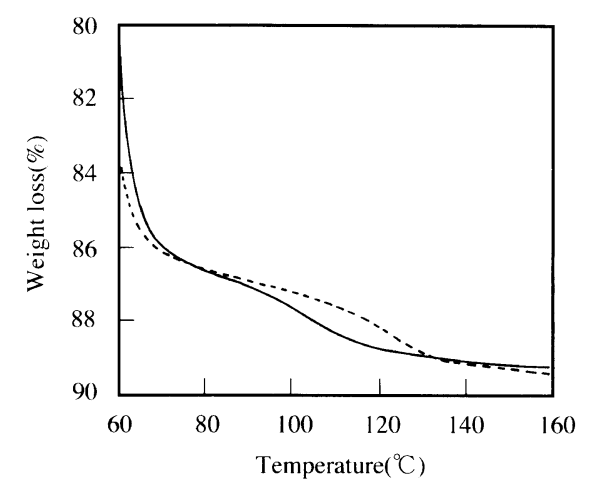

Figure 2. TG curves of SPS-xylene isomer gels: (--) p-xylene and (-) $m$-xylene.

xylene molecules from SPS crystal ( $\delta$ from) because the peak temperatures in the DSC thermograms are in agreement with the temperatures of the weight loss in TG curves of SPS-xylene gels. It is worth noting that the desorption temperatures of both xylene isomers from SPS crystal are different, respectively, although the boiling point (bp) of PX and MX are almost identical (138.4 and $139.1{ }^{\circ} \mathrm{C}$, respectively). Figure 2 shows TG curves of SPS-xylene gels. The weight loss of the sample results in the evaporation of xylene molecules which exist in SPS gel and the weight of xylene molecules seem to decrease by two step, i.e., this may suggest that there are two spaces in which xylene molecules can be located. It is known that SPS gels consist of crystalline complexes, which act as crosslinking points in the gel. ${ }^{19}$ Figure 3 shows the schematic representation of SPS gels and bold lines mean SPS chains which exhibit TTGG conformation by forming crystalline complex with solvents. The hatched areas (Region I and II) represent the spaces in which solvent molecules can be located. The solvents in Region I and II are assigned to those which swell SPS matrix, and form crystalline complex with SPS, respectively. We can presume that the first and second weight loss of xylene molecules are assigned to be located in Region I and II, respectively. The behaviors of the second weight loss of xylene molecules (i.e., the weight loss of xylene molecules complexed with SPS) seem to be dependent on the xylene isomers. The weight of PX at the second step decreases more largely 


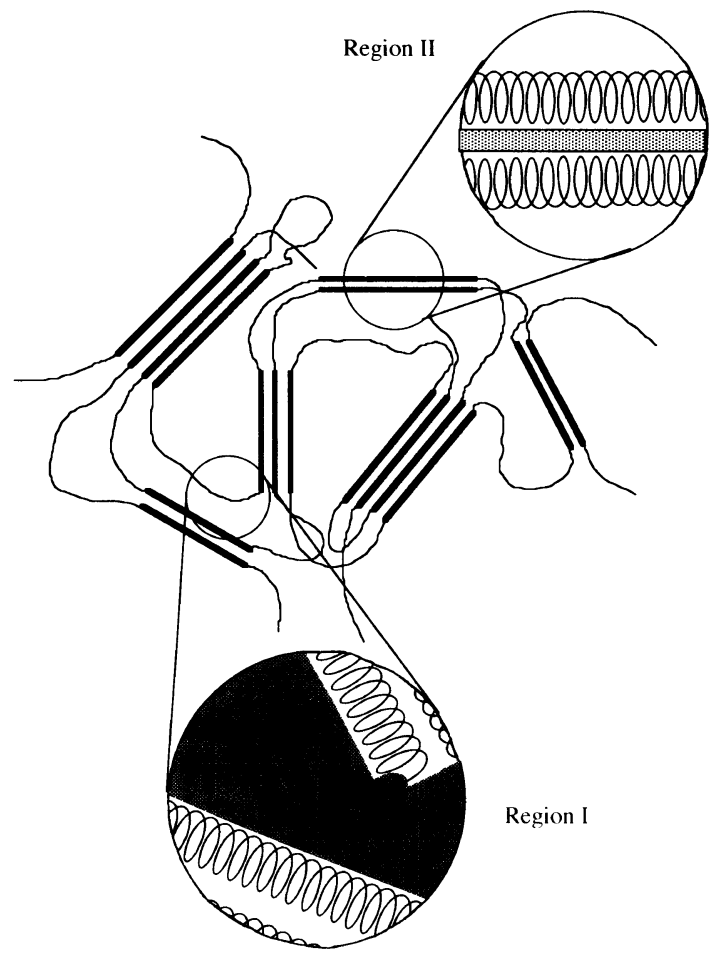

Figure 3. Schematic representation of two spaces (Region I and Region II) in which xylene molecules can be located in SPS gel.

and at higher temperature than that of MX. This result may show larger content of complexed $p$-xylene with SPS and higher thermal stability of crystalline complex of $p$-xylene with SPS. The number of moles of complexed xylene with SPS can be estimated from the second weight loss of the sample shown in the TG curve (Table II). We can conclude that PX exhibits higher ability of complex formation with SPS than MX from thermal analyses in solution system.

SPS gels consist of crystalline complexes, which act as crosslinking points in the gel. Accordingly, SPS gel formation is expected to be affected by the used solvent, i.e., ability of complex formation of the used solvent with SPS. We investigated the difference of SPS gel formation process between xylene isomers by IR measurement. The SPS chains in crystalline complex ( $\delta$ form) exhibit $2_{1}$ helix which consists of TTGG conformation, which is IR active. Figures 4 and 5 show the time dependence of IR spectra of SPS-xylene gels in $2000-700 \mathrm{~cm}^{-1}$. The absorbances of two peaks in both spectra increase with gelation time. The absorbances are assigned to TTGG conformation of SPS chains in SPS-xylene gel. The increase of TTGG conformation in both systems means the increase of crystalline complex of xylene molecules with SPS. Figure 6 shows the plot of the absorbance at $750 \mathrm{~cm}^{-1}$ vs. gelation time. These results show that the content of TTGG conformation is larger and the completion of gelation is faster in SPS-PX system. It is apparent that PX, which showed higher ability of complex formation with SPS than MX, can induce TTGG conformation largely and rapidly in SPS gel.

Isomer Effect on Xylene Sorption Behaviors of SPS Film

Our data show that PX exhibits higher ability of complex formation with SPS than MX from thermal

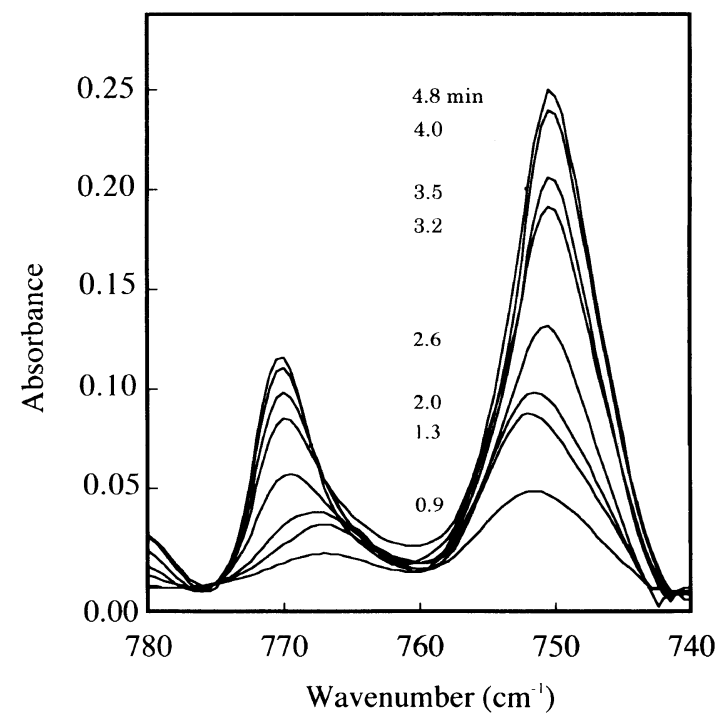

Figure 4. Time-resolved IR spectra of SPS- $p$-xylene gel.

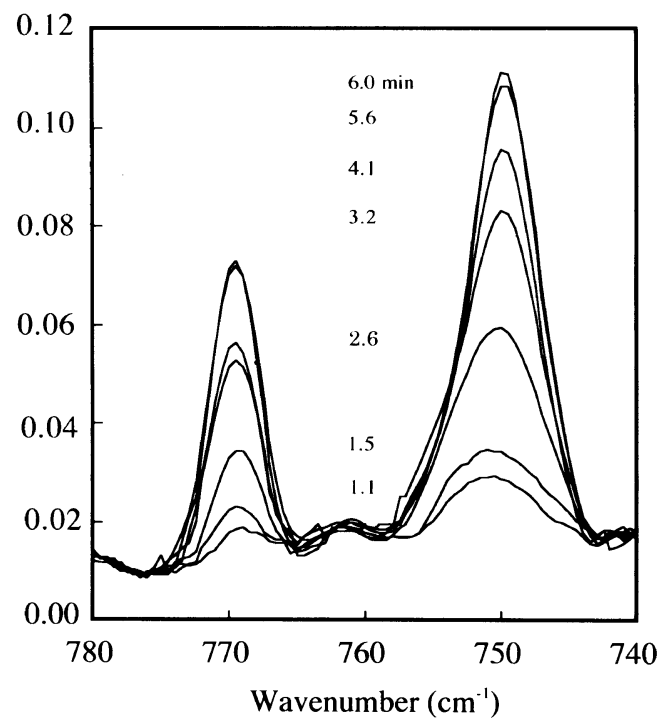

Figure 5. Time-resolved IR spectra of SPS- $m$-xylene gel.

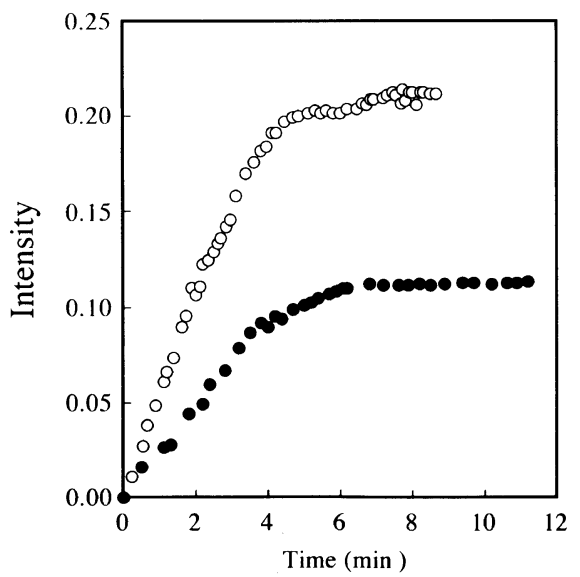

Figure 6. Time evolution of $750 \mathrm{~cm}^{-1}$ band intensity of SPS gels at $25^{\circ} \mathrm{C}$ : $p$-xylene $(\bigcirc)$ and $m$-xylene $(0)$

analysis. So we will report the isomer effect on sorption behaviors of SPS film in SPS-xylene vapor system. It is expected that the sorption behaviors of the SPS film in 
SPS-xylene vapor system may be affected by the difference of complex formation between xylene isomers because the complex formation with SPS is dependent on xylene isomers and molecular size and shape are very important factors for complex formation with SPS. It is also investigated whether a mesophase can mem-

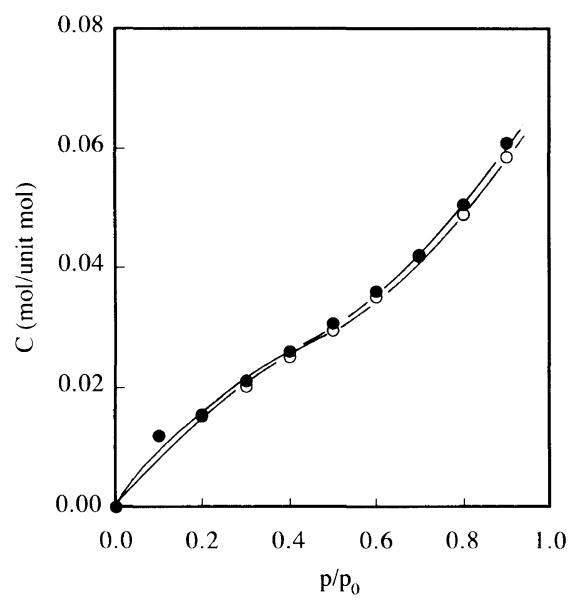

Figure 7. Sorption isotherms of $1 \mathrm{PX} 140$ (APS) at $25^{\circ} \mathrm{C}$ : $p$-xylene $(\mathrm{O})$ and $m$-xylene $(\mathbf{O})$.

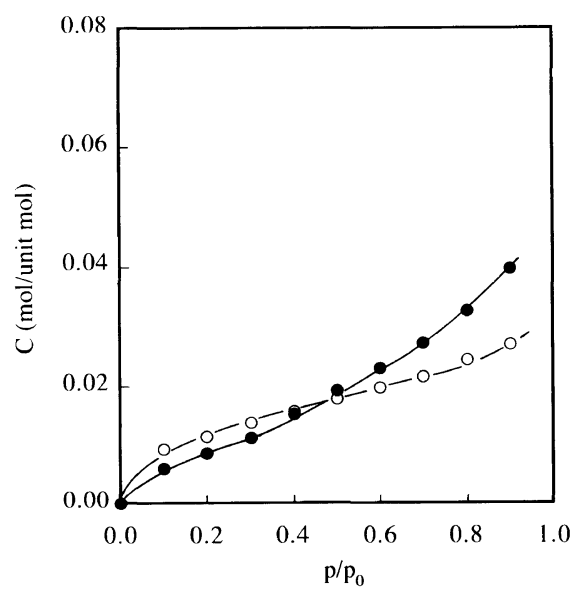

Figure 8. Sorption isotherms of $1 \mathrm{PX} 140$ at $25^{\circ} \mathrm{C}$ : p-xylene $(O)$ and $m$-xylene ( orize molecular size and shape of the cast solvent.

Figure 7 shows $\mathrm{PX}$ and $\mathrm{MX}$ vapor sorption isotherms of 1 PX 140 (APS) at $25^{\circ} \mathrm{C}$. The two sorption isotherms in the APS film showed a normal good solvent type and could be described by Flory-Huggins thermodynamics and the difference of the sorption behaviors between PX and MX is not almost observed, because the interaction with APS is almost the same between both xylene isomers. Figure 8 shows PX and MX vapor sorption isotherms of $1 \mathrm{PX} 140$ at $25^{\circ} \mathrm{C}$. These sorption data are not corrected based on the crystalline content of the SPS film. PX and MX sorption isotherms showed sigmoid-type isotherms and were concave toward the activity axis at low activity as reported on sorption behaviors in SPS-toluene vapor system in a previous paper, ${ }^{17}$ but both xylene isomers sorption behaviors are different. Sorption amount of PX is larger at low activity, but smaller at high activity than that of MX. We performed thermal and spectroscopic analyses of the SPS film which was prepared by casting toluene solution and then annealed at lower temperature than conformation transition temperature $\left(190^{\circ} \mathrm{C}\right)$ in vacuum suggested that the films exhibited an ordered conformation but a lack of crystalline regularity in a previous paper. ${ }^{16}$ This structure was defined as a mesophase. From $\mathrm{CO}_{2}$ sorption measurement, it was apparent that the films contained many solvent desorption sites as nanopores in the mesophase (Figure 9). From these results, we suggested that the concaveness of toluene vapor sorption isotherm of the SPS film toward the pressure axis at low activity is due to the preferential sorption of toluene vapor into the solvent desorption sites in the mesophase. ${ }^{17}$ The SPS films prepared by casting from PX solution consist of $\delta$ form (crystalline complex between SPS and PX) and a mesophase can be formed by annealing the PX cast SPS films under appropriate conditions such films obtained by annealing the toluene cast films. ${ }^{16}$ From these results, the concaveness of the sorption isotherm toward the activity axis at low activity might be due to the preferential sorption of PX into the mesophase containing many desorption sites of PX, which is a cast solvent (Figure 10). The larger sorption amount of PX may suggest that
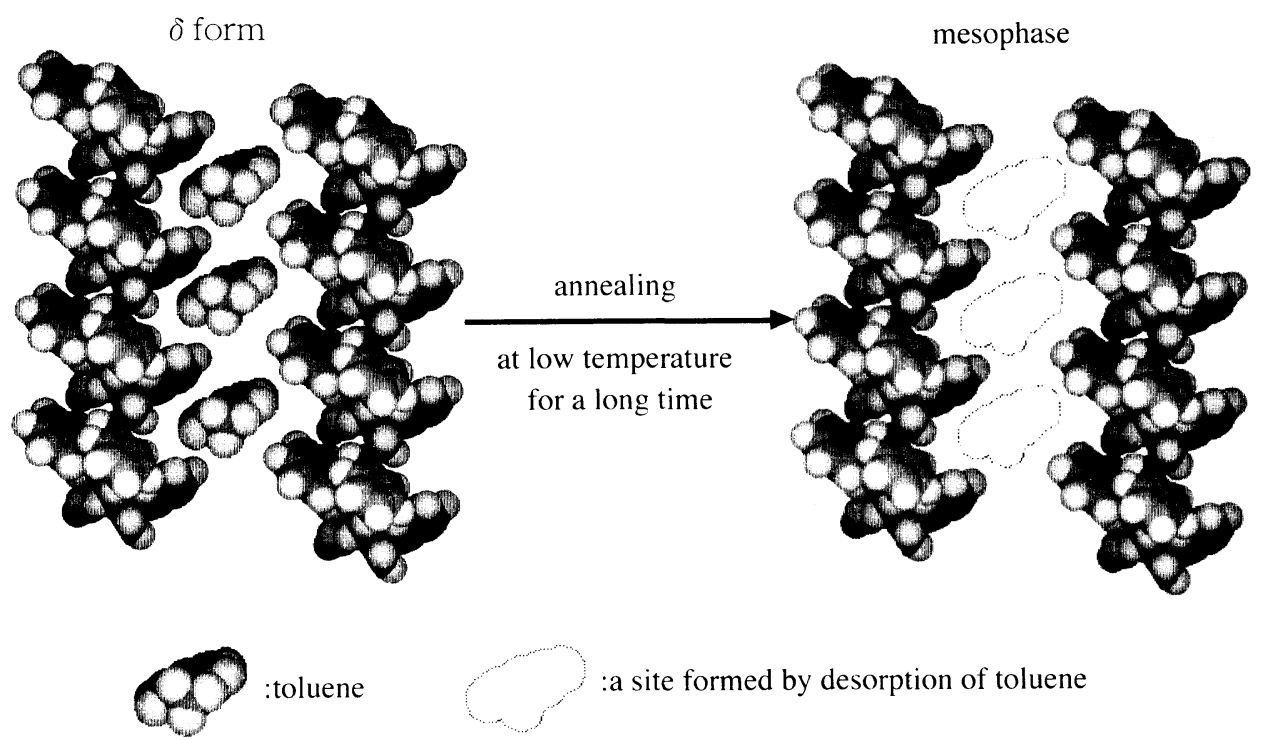

:a site formed by desorption of toluene

Figure 9. Schematic representation of reorganization from $\delta$ form to mesophase in SPS-toluene system 
mesophase

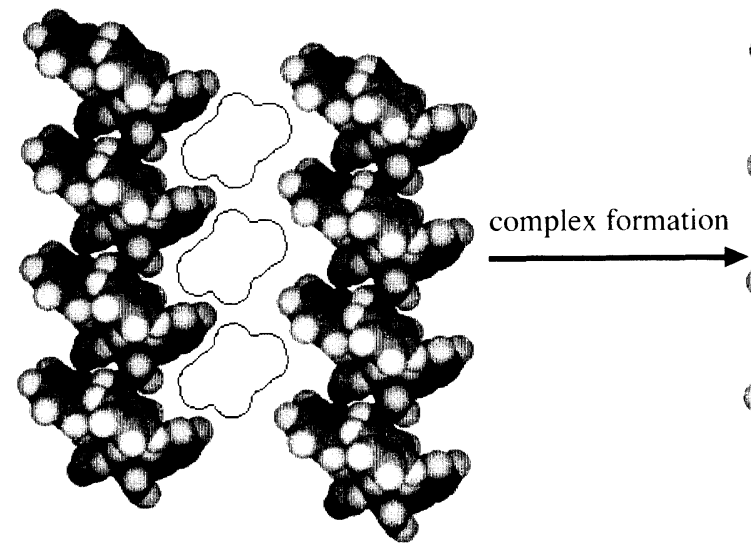

$\delta$ form
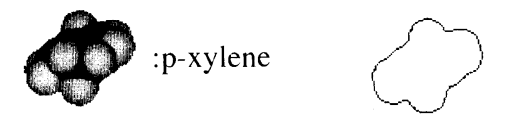

:a site formed by desorption of p-xylene

Figure 10. Schematic representation of complex formation from mesophase to $\delta$ form in SPS- $p$-xylene system

Table II. The number of moles of complexed solvent with SPS estimated from TG measurement

\begin{tabular}{cc}
\hline Sample & Mol (complexed solvent)/Mol (SPS unit) \\
\hline PX-SPS gel & 0.024 \\
MX-SPS gel & 0.020 \\
\hline
\end{tabular}

there is a possibility that PX desorption sites in a mesophase can memorize size and shape of PX and the desorption sites act as effective sorption sites for PX molecules at low activity.

It is well known that sorption of vapors and liquids into polymeric films can cause significant plasticization resulting in substantial decreases in the glass transition temperature $\left(T_{\mathrm{g}}\right)^{22,23}$ If the reduction of $T_{\mathrm{g}}$ of used crystalline polymer is large enough to put the system in the crystallization temperature region, the polymer chains rearrange themselves into a lower free energy state, ${ }^{24,25}$ i.e., plasticization of penetrant molecules can induce crystallization of crystalline polymers. In SPS xylene system, it is expected that plasticization of $x y-$ lene molecules can induce crystallization (formation of crystalline complex) of SPS film at high activity. In general, crystal formation decreases sorption amount of penetrant molecules because an amorphous part in the SPS film decreases by complex formation and expansion of polymer matrix by sorption of penetrant molecules is depressed. It is clear from Table II that PX exhibits higher ability of complex formation with SPS than MX in solution system. Accordingly, we insist that the sorption amount of PX at high activity decreases because of higher ability of complex formation with SPS.

\section{CONCLUSIONS}

We reported the isomer effect on complex formation in SPS-xylene system. Thermal behaviors in solution system were investigated to get information on the isomer effect of xylene on ability of complex formation with SPS. The obtained results showed that PX exhibited higher ability of complex formation with SPS than $m$ xylene. From these results, we proposed that the molecular size and shape of PX might fit the cavities formed by two adjacent phenyl groups of SPS chains. The SPS gel formation process was also affected by ability of complex formation of xylene isomers with SPS. We also reported the xylene sorption behaviors of the annealed SPS film containing a mesophase which could memorize molecular size and shape of the used cast solvent. The difference of the sorption behaviors of the APS film was not observed between xylene isomers, but the xylene isomer sorption isotherms of the SPS film were dependent on xylene isomers because of the unique morphology of the SPS film and the complex formation of xylene with SPS. Sorption amount of PX was larger than that of MX at low activity, but smaller at high activity, which may result in the preferential sorption of PX into a mesophase at low activity and the decrease of sorption sites by complex formation of PX with SPS at high activity. From the obtained results, we suggested the possibility that the sorption sites in the mesophase could memorize molecular size and shape of the used cast solvent. SPS films will be very useful for isomer separation films because of the unique morphology of SPS films and the difference of ability of complex formation of isomers with SPS, different from other isomer separation methods by the usual films based on the difference of diffusivity among isomers.

Acknowledgment. The authors gratefully acknowledge partial financial supports from the Grant-in-Aid for scientific research from the Ministry of Education, Science, Sports and Culture of Japan(10123214) and the Petroleum Energy Center (PEC) subsidized from Ministry of International Trade and Industry, and Tokyo Ohka foundation for the promotion of Science and Technology.

\section{REFERENCES}

1. W. T. Winter and A. Sarko, Biopolymers, 13, 1461 (1974). 
2. H. Kusuyama, N. Miyamoto, Y. Chatani, and H. Tadokoro, Polym. Commun., 24, 119 (1983).

3. J. Watanabe, Y. Sasanuma, A. Endo, and I. Uematsu, Polymer, 25, 698 (1984).

4. A. E. Tonelli, Polymer, 35, 573 (1994).

5. A. Kawaguchi, Polymer, 35, 3797 (1994).

6. N. Vasanthan, I. D. Shin, and A. E. Tonelli, Macromolecules, 29, 263 (1996).

7. Y. Chatani, Y. Shimane, Y. Inoue, T. Inagaki, T. Ishioka, T. Ijitsu, and T. Yukinari, Polymer, 33, 488 (1992).

8. Y. Chatani, Y. Shimane, Y. Inoue, T. Inagaki, T. Ijitsu, T. Yukinari, and H. Shikuma, Polymer, 34, 1620 (1993).

9. Y. Chatani, T. Inagaki, Y.Shimane, and H. Shikuma, Polymer, 34, 4841 (1993).

10. T. Roels, F. Deberdt, and H. Berghmans, Macromolecules, 27, 6216 (1994).

11. C. Daniel, A. Menelle, A. Brulet, and J.-M. Guenet, Polymer, 38, 4193 (1997).

12. M. Kobayashi, T. Yoshioka, T. Kozaka, K. Tashiro, J. Suzuki, S. Funahashi, and Y. Izumi, Macromolecules, 27, 1349 (1994).

13. M. Kobayashi, T. Yoshioka, M. Imai, and Y. Itoh, Macromolecules, 28, 7376 (1995).
14. C. Daniel, M. D. Deluca, J.-M. Guenet, A. Brulet, and A. Menelle, Polymer, 37, 1273 (1996).

15. J.-M. Guenet, TRIP, 4, 6 (1996).

16. K. Tsutsui, Y. Tsujita, H. Yoshimizu, and T. Kinoshita, Polymer, 39, 5177 (1998).

17. K. Tsutsui, T. Katsumata, Y. Yamamoto, H. Fukatsu, H. Yoshimizu, T. Kinoshita, and Y. Tsujita, Polymer, in press.

18. G. Sauerbrey, Z. Phys., 155, 206 (1959).

19. H. Itagaki and Y. Nakatani, Macromolecules, 30, 7793 (1997).

20. S. Coffey, Ed., "Rodd's Chemistry of Carbon Compounds," 2nd ed, Vol. III, Part A, Elsevier, New York, N.Y., 1971, p 179.

21. T. E. Daubert and R. P. Danner, "Physical and Thermodynamic Properties of Pure Chemicals," Hemisphere Publishing Corp., New York, N.Y., 1989.

22. G. A. Bernier and R. P. Kambour, Macromolecules, 5, 393 (1968).

23. R. P. Kambour and C. L. Gruner, J. Polym. Sci. Polym. Phys. Ed., 16, 703 (1978).

24. W. R. Moore and C. L. Gruner, Polymer, 2, 315 (1961).

25. P. J. Makarewicz and G. L. Gruner, J. Polym. Sci., Polym. Phys. Ed., 16, 1559 (1978). 